\title{
Presenting the NapoNet: Developing Global Competencies through Commu- nications Technology in the Peruvian Amazon
}

\section{Miss Christie Ritter, The University of Colorado at Boulder}

Christie Ritter is a Junior in Environmental Engineering with a focus in Engineering for Developing Communities at the University of Colorado at Boulder.

\section{Dr. Alan Rolf Mickelson, University of Colorado, Boulder \\ Dr. Daniel Knight, University of Colorado, Boulder}

Daniel W. Knight is the engineering assessment specialist at the Integrated Teaching and Learning Program and Laboratory. He holds a BS in psychology from Louisiana State University, and an MS degree in industrial/organizational psychology and $\mathrm{PhD}$ degree in counseling psychology, both from the University of Tennessee. Prior to joining the University of Colorado at Boulder, he gained extensive experience in assessment and teamwork in an engineering education context through the development and evaluation of a team facilitation training course for engineering undergraduate students. Dr. Knight's research interests are in the areas of retention, program evaluation and teamwork practices in engineering education. His current duties include the assessment and evaluation of the ITL Program's hands-on undergraduate courses and K-12 engineering outreach initiatives.

\section{Jared Leventhal, University of Colorado at Boulder}

Jared Leventhal is currently an undergraduate student at the University of Colorado at Boulder pursuing a B.S. in Civil Engineering as well as a B.S. in Business Administration with an emphasis in Marketing. $\mathrm{He}$ is an active student researcher as part of the NapoNet team.

\section{David Espinoza, University of Colorado at Boulder}

David Espinoza is an electrical engineer with experience in the design of open-source based wireless routers and management of deployments of wireless networks in rural areas of Peru. He graduated from the masters program in Telecommunications and Engineering Management and joined the $\mathrm{PhD}$ program in Telecommunications at CU-Boulder. His professional interests are in Information and Communication Technologies for Development and Sustainable Roll Out of Telecommunications Services for Rural Areas of Developing Countries. He has volunteered in the NapoNet project, deployed in the Peruvian Rain Forest, as technical, logistic and field deployments advisor under the supervision of Professor Alan Mickelson since 2008. 


\section{Presenting the NapoNet: Developing Global Competencies through Communications Technology in the Peruvian Amazon}

Abstract

Since its beginning in 2009, the NapoNet Program has provided an opportunity for undergraduate, graduate, and postdoctoral students to obtain technical knowledge and skills in a wide variety of aspects of Global Engineering for community development. The NapoNet team includes students from various backgrounds of study, and has partnered with professionals and academic entities in South America, mostly in Peru. The construction of the network began with a project driven by the United Nations-supported Global Fund for HIV, Tuberculosis, and Malaria to install a wireless network along the Napo River to provide a means of communication for villages along the river and primarily connect health posts. From this, the Grupo de Telecomunicaciones Rurales (GTR) installed transceivers along $445 \mathrm{~km}$ of dense, canopied rainforest along the Napo River. The University of Colorado at Boulder began this partnership to find ways to use the network to improve health and education in the Napo region.

The NapoNet team represents a unique mix of backgrounds and technical abilities, which lends to an educational experience for all involved. Technical knowledge of the wireless towers is required, but several other disciplines have emerged as important to the progress of the team. One of the imperative aspects of the NapoNet project is an acute cultural awareness. The network has the possibility of serving people from several backgrounds, including descendents from Spanish colonies in Peru, as well as several unique indigenous tribes that call the Napo region home. Another perspective approached by this project is an intentional consciousness of the river and rainforest ecology. The commerce, as well as a general way of life on the Napo River, depends heavily on the river as well as the plants and animals that naturally inhabit it. Due to the inherent importance of the environment, the NapoNet team has provided the opportunity to consider its role in communications technology as well as in culture. An interest in the commerce of the region creates the need for an understanding of business as well.

Global engineering for community development represents the foundation of the NapoNet project. The intricate intersection of communications, culture, ecology, and commerce are the applications within this greater purpose. Because these applications cover such a broad scope, the NapoNet team has invited members of varied backgrounds to participate in research together. Undergraduates and graduate students participate in projects with backgrounds including Information and Communications Technology, Education, Anthropology, Psychology, Environmental Design, Business, and several branches of engineering including Civil, Environmental, and Electrical Engineering as well as Engineering Physics. Technical projects with a global prominence have compelled each of these team members to step outside of their own areas of study and expertise to learn in a much more expansive and interdisciplinary sense. This in turn allows them to understand their own contributions more completely. Consequently, the learning achieved as a function of the NapoNet prompts members to stretch their abilities, create wider boundaries of comfort in approaching technical solutions, and accomplish an improved overall idea of what it is to participate in a global context. Data will be shared related to student learning experiences, the development of global competencies, and impact on the participating communities. 
Introduction

Global development has been a topic of interest as of late, but the focus has shifted from whether or not development is necessary to how development can be carried out in the most sustainable and responsible manner. Several arms of global development exist, including drinking water, education, transportation, energy, and telecommunications ${ }^{1}$. The argument of which of these topics is most important continues to occur on a large scale in the academic world. The NapoNet project based at the University of Colorado at Boulder (CU) does not serve to answer this question, but instead, provides an awareness of these arms and their interdependent importance in the realm of Global Engineering. The Napo River region in Peru offers an ideal perspective on these different facets working together, as well as a look into appropriate engineering solutions.

One of the major characteristics that renders the NapoNet project unique is the isolated location of the network. The wireless towers that comprise the network are located in villages along the Napo River. A dense rainforest canopy makes microwave penetration (and therefore communications technology) as well as basic transportation to and from these villages difficult ${ }^{2}$. Boat travel is the only way to move from village to village. These constraints are present due to the geography of the region and provide a unique opportunity to understand appropriate engineering solutions. Members of the NapoNet team have, out of necessity, learned to take these constraints into account in order to fulfill project requirements.

The interdisciplinary nature of the NapoNet team also presents a unique environment for learning in a global and technical sense. Members of the team come from varying backgrounds, including Information and Communications Technology, Education, Anthropology, Psychology, Environmental Design, Business, and several branches of engineering including Civil, Environmental, and Electrical Engineering as well as Engineering Physics. The team blends a variety of backgrounds with an inherent variety of cultures in the partnerships in Peru to function cohesively. As a result, members of the team must adapt to articulating new ideas or information in a clear and succinct matter so that every member of the team understands. Different disciplines and cultures approach communication, technology, and problem solving in unique ways and it is imperative that these differences be addressed so that the overall team can understand a central idea or objective. Communicating in this manner also consequentially compels team members to approach problem solving in global engineering from several perspectives, instead of only their individual background. The more technically trained team members learn to apprehend some of the sociological impacts of technical solutions, and the team members with stronger backgrounds in social science learn an engineering approach to problem solving. The global nature of the NapoNet project also provides for the exchange of cultural knowledge and awareness. These crossovers in background create a more well rounded project, team, and finally, individual.

NapoNet: Partners, Scope, and History

The NapoNet project is a collaborative effort between faculty at the University of Colorado at Boulder, the Grupo de Telecomunicaciones Rurales ${ }^{3}$ (GTR) of the Pontificia Universidad Catolica del Peru (PUCP) ${ }^{4,5}$, the Centro de Salud de Santa Clotilde (CSSC), and the Government of the State of Loreto, Peru. The focus of NapoNet is to make better use of the 
bandwidth of the Pamafro WiFi Long Distance network (WiLDNet) in order to serve the people of the middle and upper Napo River of Peru. The most cost effective technology with which to implement jungle communications given that towers are necessary to rise above the forest canopy is that of WiFi Long Distance ${ }^{6}$. Indeed, the end to end information bandwidth of the 445 $\mathrm{km}$ long WiLDNet is estimated to be between 1 and $3 \mathrm{Mbs}$, a bandwidth much greater than that necessary for transmitting patient information and logistical telephone calls between the health posts, but one too small to transmit full internet service to all those on the network. The NapoNet project focuses on developing, implementing and evaluating bandwidth efficient methods for bringing communication services to those who live in the villages along the network.

The NapoNet Project was initiated in February of 2009. The project was suggested to NapoNet team members by faculty members of the University of Amazon of Iquitos, Peru, and public health and government officials of the State of Loreto, Peru. The long term goal of the project is to imaginatively use the bandwidth of the Pamafro WiFi Long Distance network (WiLDNet) to bring services (health, education and commerce) to villagers of the middle and upper Napo River basin without compromising the original health care purpose for which the network was proposed, funded and constructed ${ }^{7}$. NapoNet (the Pamafro Network) lies in the province of Maynas of the state of Loreto, Peru. Loreto is the largest but poorest state of Peru ${ }^{8}$. NapoNet extends $480 \mathrm{~km}$ from Iquitos, at the junction of the Amazon and Napo River, along the Napo River northeast to the Ecuador border at Cabo Pantoja. Since 2009, the NapoNet team has undertaken several trips per year focused on expanding program initiatives that include: installation and maintenance of network components, water quality testing, education subnetwork deployment, commerce applications, and mixed-method community assessments.

\section{Educational Subnetwork}

Several trips to the Peruvian Rainforest reached the middle Napo River basin communities and involved meetings with relevant community leaders and members, participatory group interviews and a number of experiments that would determine the best approaches to network augmentation. The findings from the meetings and interviews revealed the people's basic services priorities: 1) health care, 2) education, 3) economic activities, and 4) vocational training. Since the health care area had already been covered by the original team which deployed the network, the CU team decided to support education initiatives. The pilot project consisted of installing an educational subnetwork in the schools that could be dedicated to distance education. Students who traveled to the Napo Region had the chance to interact with the locals, learn from and teach the locals, understand the local culture, and exchange daily experiences (See Figure 1).
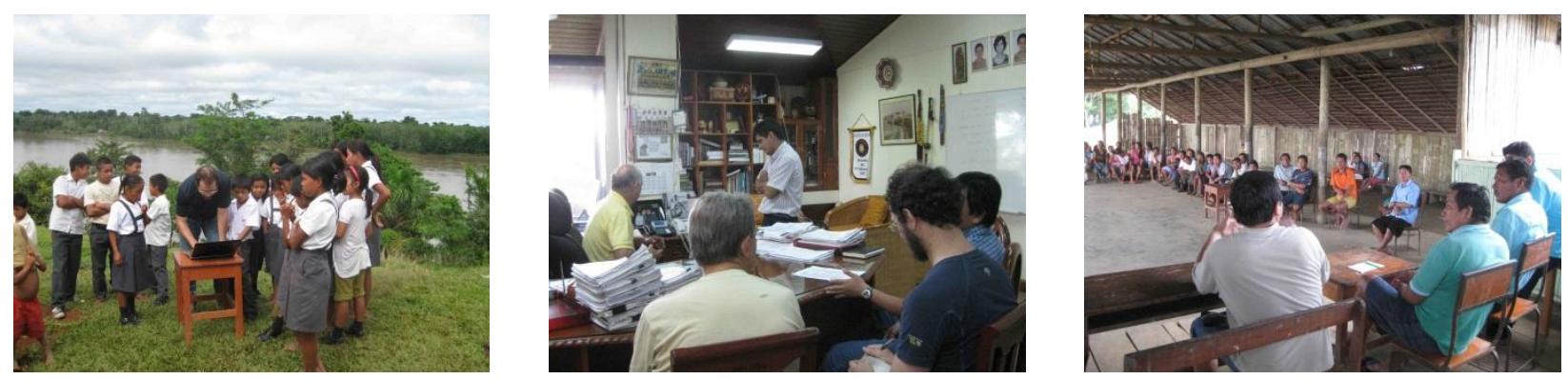

Figure 1: L-R (1) installing computer scripts to control transmission and routing of packets to 
Voice over Internet Protocol (VoIP) phones; (2) meeting with the Governor of the State of Loreto, Peru in a working session; (3) conducting focus groups for qualitative analysis based assessments.

On the technical side (Figure 2), engineering students carried out maintenance tasks on the telecommunications systems at each community they visited, and deployed the distance educational pilot in a few selected communities. The maintenance tasks were carried out on radios and antennas, batteries and solar panels, and PCs. Most of the computer science and telecommunications design and testing were performed at a lab at $\mathrm{CU}$ prior to the trip.
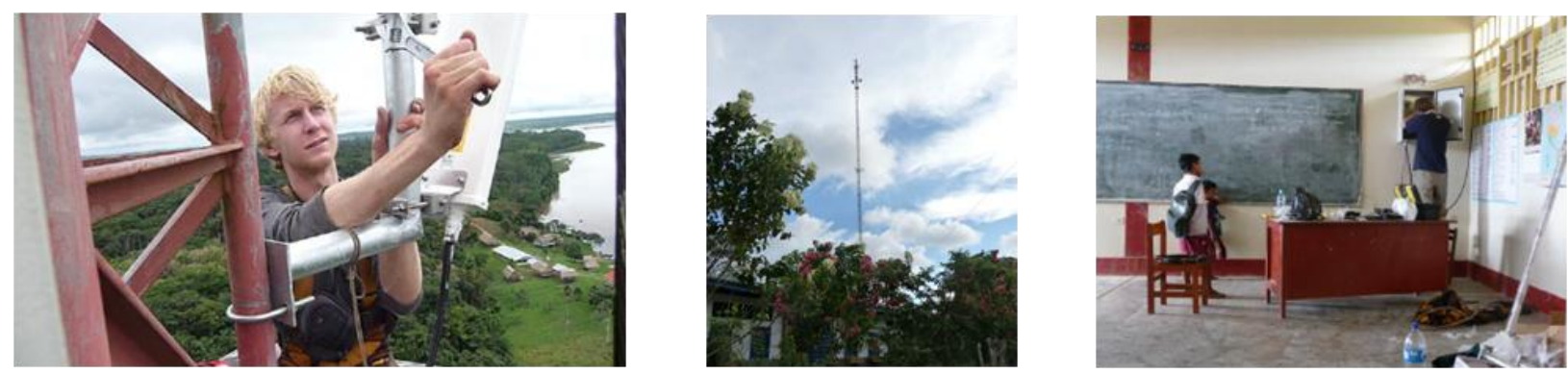

Figure 2: L-R (1) undergraduate researcher working on a tower; (2) network tower in Santa Clotilde; (3) undergraduate researcher installing a wireless router in the primary school in Campo Serio.

Additional learning came from carrying out the logistics for the deployments of the educational pilot and working with technical and non-technical partners from Peru. Some lessons learned were to run the project under flexible schedules, to deal with unexpected events, to readjust the work schedule to still try to meet estimated deadlines, and to encourage motivation of partners to perform well. Since most of the supplies were purchased in Lima, capital of Peru, and delivered to the communities in the Napo, coordinating the tracking and delivery of the equipment on time was an educational experience in and of itself.

Traffic on the NapoNet

The NapoNet project provided a variety of opportunities for undergraduate and graduate students to participate in a real-world telecommunications project. Students started working on re-creating the NapoNet network in a CU laboratory to better understand how it works and improve its efficiency. The laboratory equipment included the integration of Linux-based wireless routers. These integrated wireless routers hosted advanced applications such as dynamic and static routing protocols, VoIP telephone servers, and configurable WiFi card drivers. Additionally, Asterisk servers were installed in each of the wireless routers in the Napo river network which allowed the router to send logs containing phone call information to a server on the CU campus, which is subsequently used for data mining. These logs are received on a daily basis and each file contains 18 data points per phone call. As part of this effort, students use the database of the logs for purposes of extracting important statistics that can indicate trends in the usage of the phones and where/how the network could be improved.

Undergraduate engineering researchers worked on network dynamics through analysis of traffic recorded in the database of the logs. They collaborated with graduate students in writing 
data mining Python scripts that now allow for the ability to look at call density through the network over arbitrary periods of time. This has led to a broader understanding of bandwidth capabilities and network dynamics. The students worked on hardware and software simulation of the network, that is, of replicating two nodes of the network in a lab at CU to understand network problems such as dropped calls. As a result of this research, Android $4 \mathrm{G}$ phones are now being used over the network.

The team has been receiving, with some regularity, daily downloads of the log data. Data mining efforts are evolving and error bars are gradually shrinking, as there is a better understanding of what the data is, what is repeated, what is spurious, and what is mainstream reliable. Knowledge of the traffic is important as there are limits to the capacity of such a network. Given a calculated capacity of 100 calls simultaneously, there is available bandwidth for further development and expansion of the existing network into the domains of health, education, and commerce.

\section{Commerce Project}

One of the areas currently under expansion by the NapoNet team is the role of the agricultural economy along the river. Community surveys revealed a great disparity in the income of the farmers along the Napo River and the buyers in the market in the city of Iquitos. Farmers only receive a small price for their product, and middlemen with boats purchase and transport these crops downriver. Once they reach the market, the middlemen sell these crops for a much higher amount, but still do not make a substantial living due to logistical inefficiencies with their boat operation and high gasoline prices. Data collected in the communities showed that there is a markup of up to $1000 \%$ from farmer to buyer, and that farmers could potentially make more income than they currently do.

Looking at the intersection of telecommunications and economy, it became an objective of the team to understand how telecommunications technology could be used to transfer information about the market to stakeholders. As a result, the farmers would have more information available to them about what their crops are worth, and boat operators or transporters could plan more organized routes to save gas and completely fill up their boats with each trip.

The Rural Trade Communications venture is under development by the NapoNet team to address this disparity. Using the wireless network and smartphone technology, the farmers can be put in contact with the buyers through leveraging communication networks. In a global engineering context, technology and organizational methods must be adapted to fit the culture and attitudes of the people intended to be served. The NapoNet team presents a tangible way to learn this in the context of truly creating a social enterprise.

The development of the Rural Trade Communications venture has also provided an avenue for the members of the NapoNet team to learn about the role of business in engineering and global development. A constant goal and perspective of the team is to act with long term sustainability in mind. This is the primary reason that the proposed solution to the disparity between farmer and buyer on the Napo River evolved to the creation of a venture. A social venture operates under the idea that the customers and beneficiaries must buy into the venture, 
thereby creating an investment into the organization or idea ${ }^{9}$. In comparison to only handing out aid in a low resource region, the creation of a business has shown more promise in development situations ${ }^{10}$.

Program Assessment

Assessment of the NapoNet program follows a mixed-methods approach with quantitative and qualitative methods implemented for evaluating program goals and objectives. Assessment methods were carried out against the following program goals:

Goal 1: Develop the NapoNet telecommunications infrastructure to support program initiatives, Goal 2: Integrate the NapoNet into the Napo River communities in Peru, Goal 3: Provide opportunities for CU student development.

Goal 1 is assessed via the network traffic monitoring system developed at CU. Careful analysis of the data logs continues to monitor the viability of the network. Analysis of the data has revealed the availability of additional bandwidth to further NapoNet Program initiatives. Goal 2 is assessed via focus groups and interviews with partners in Peru including people living in the villages, government officials, and members of local organizations. Results from these assessments have determined the eagerness of Peruvian partners to collaborate with CU to improve health, education, and commerce in the Napo region through the use of telecommunications technology. Assessments have also identified a downside of introducing this technology, that being a stratification of the village communities into those who have internet access and those who do not. Those who have access, including health and education professionals who are often foreign, now enjoy greater status in the community while those without internet access feel disenfranchised. Additional assessments are ongoing to better understand this stratification and ameliorate its effects on the community.

Goal 3 is assessed by surveying students who have participated in the program. Twelve students were queried about the impact of the program on the development of important global competencies such as an understanding of the role of engineering in global development, a better understanding of interdisciplinary perspectives, and the impact of the program on interest in engaging in future global development projects. Results were obtained from nine students (75\% return rate). The majority of respondents (80\%) participated during their sophomore or junior year in school and majored in engineering $(88 \%)$. Quantitative survey results can be viewed in Table 1. Students rated each outcome on a five-point Likert type scale with level agreement calculated from the percentage of students who indicated "agree" or "strongly agree for each item. Each question also allowed for open-ended comments.

Results indicate that the highest level of agreement on NapoNet program outcomes is for the impact on understanding of global development, the unique nature of the program compared to other educational opportunities, and the development of interest in future projects like NapoNet. Student comments support these results with one student reporting, "I would say the biggest thing I was taught from NapoNet is how complex development work truly is. I had known that there were a million different aspects involved in getting a project to work successfully, but it wasn't until I was involved in the work itself that I truly understood the issues that can arise in any project." Another student commented, "Going on the trip taught me that 
Table 1: Level of Agreement on Student Outcomes for the NapoNet Program

\begin{tabular}{|l|c|}
\hline \multicolumn{1}{|c|}{ Program Outcomes } & $\begin{array}{c}\text { Level of } \\
\text { Agreement }\end{array}$ \\
\hline Understanding of global development & $88.9 \%$ \\
\hline Sparked personal interest in additional projects of this nature & $88.9 \%$ \\
\hline Provided a unique student experience at CU & $88.9 \%$ \\
\hline Interdisciplinary nature contributed to personal development & $77.7 \%$ \\
\hline Met personal expectations & $77.8 \%$ \\
\hline Understanding of engineering in a global sense & $66.6 \%$ \\
\hline
\end{tabular}

working in development is never a straightforward engineering project and it is more about understanding the community than it is about engineering."

Regarding the unique nature of NapoNet, one student commented, "This project was so different from all the others that I was involved in at CU that I'm not really sure how to compare them. It gave me the chance to interact in a constructive way with a culture entirely different from my own and allowed me to apply my learning to a problem that I had never really thought of before." Another student remarked, "NapoNet has been the most realistic project that I have worked on, as it has been necessary to consider consequences, opportunities, challenges, and action plans. I have learned how to ask better questions, find pertinent resources, realize constraints, and plan for the future.

With respect to future interest, "I have since become the president of Engineers Without Borders USA CU chapter, joined the ICTD program in ATLAS, and become a consultant and student for MC-EDC (all global development organizations). The NapoNet project was my first international engineering for development project, and I owe a lot to that." Another commented on developing interest, "It has made me more interested in the social sciences aspects of working in communities whose needs and desires might not be fully understood from the perspective of an American engineer.

The lowest level of agreement for NapoNet program outcomes is for the impact on understanding engineering in a global sense. One student commented, "The NapoNet project is localized to one district within Peru, and it is difficult to see how the project impacts the people that live outside of this sphere very much." This comment points to an area for improvement in the NapoNet curriculum about increasing student understanding of engineering in a global context. Students could be assigned readings and case studies to augment the mostly applied nature of the project. One note of caution for interpreting the student surveys results is the small size $(n=9)$ of the sample.

Summary and Conclusion

Since its inception in 2009, the NapoNet program has afforded participants and partners with the opportunity to expand communications and internet into rural regions along the Napo River in Peru and understand the impact of this technology on the communities served by the program. While global engineering for community development is the foundation for this program, solving the problems inherent in this endeavor has led to the incorporation of partners 
from other disciplines which has led to a very interdisciplinary flavor for the project.

A process including assessment, research, and development has led to a steady improvement of the technological capabilities of the network as well as a deeper understanding of the impact of these technologies on the villages along the Napo River. An initial focus on health services has been expanded to focus on improved education and commerce along the river. In addition, a steadily growing cohort of students have become involved in the program and they report the NapoNet project is a very unique academic experience at CU that taught them a great deal about the excitement and difficulties inherent in global development. Many of the students involved were motivated to pursue future projects of this kind. Future directions along the Napo include efforts to secure federal funding to further build out the network and to better understand the social stratification caused by the introduction of the technology.

\section{References}

[1] United Nations Millennium Development Goals. http://www.un.org/millenniumgoals/.

[2] A. R. Mickelson, Q. Lv, L. Shang, and S. R. Sterling. "A 2009 NapoNet video". http://www.youtube.com/watch?v=X7t71DDNOnY.

[3] GTR|Grupo de Telecomunicaciones Rurales: Rural Telecommunications Group. http://gtr.telecom.pucp.edu.pe/.

[4] Pontificia Universidad Catolica del Peru: The Catholic University of Peru. http://www.pucp.edu.pe/content/index.php.

[5] Publicaciones|GTR: Publications. http://gtr.telecom.pucp.edu.pe/publicaciones.

[6] R. Patra, S. Nedevschi, S. Surana, A. Sheth, L. Subramanian, E. Brewer. WiLDNet: Design and Implementation of High Performance WiFi Based Long Distance Networks. USENIX NSDI, April 2007.

[7] A. R. Mickelson, Q. Lv, L. Shang, and S. R. Sterling. The NapoNet University of Colorado at Boulder Outreach Site. http://outreach.colorado.edu/programs/details/id/363.

[8] D.E. Newman and M.C. Shapiro. Obstacles faced by general practitioners in Loreto Department, Peru in pursuing residency training. Abstract in Rural Remote Health. April 2010

[9] A. R. Mickelson, J. Leventhal, C. Ritter, M. Lacy. Rural Trade Communications. http://outreach.colorado.edu/programs/details/id/363.

[10] A.R Mickelson, J. Leventhal, C.Ritter, M. Lacy. NCIIA Rural Trade Communications. http://www.youtube.com/watch?v=xlaSoOasQNQ\&feature=youtu.be. 